\title{
Extraction of Face and Palmprint Features based on LBP, HOG and Zernike Moments
}

\author{
Akram Alsubari \\ School of Computer Sciences, \\ North Maharashtra University, \\ Jalgaon, India
}

\author{
R. J. Ramteke \\ School of Computer Sciences, \\ North Maharashtra University, \\ Jalgaon, India
}

\begin{abstract}
This paper describes the recognition of multimodal biometric systems based on the face and palmprint features. The common feature extraction technique between the palmprint and face recognition system is the Local Binary Pattern. In the palmprint recognition, the region of palm was extracted form the entire hand. The Histogram of Oriented Gradient (HOG) and Local Binary Pattern (LBP) were used to extract the features of palm. The Zernike moments and LBP were used to extract the features of face. The features of palm and face were integrated as feature vector. To evaluated the accuracy of the system, different classifications were applied such as SVM, KNN, Linear Discriminant. This experiment was performed on the ORL and CASIA database. The proposed system was tested on the said database and found to be satisfactory.
\end{abstract}

\section{General Terms}

Pattern Recognition, Multi-modal Biometrics, Features Extraction.

\section{Keywords}

LBP, HOG, Zernike Moments, ROI, SVM, KNN.

\section{INTRODUCTION}

The recognition system which it's acquires biometric information from many sources for the same person to determine the identity of a person known as the multibiometrics system. Any piece of evidence can be independently used to recognize a person is called a source of biometric information [1]. The multimodal biometrics are combining more than one modalities of biometrics to improve the recognition accuracy. Biometric systems are becoming popular as a measure to identify human being by measuring one's physiological or behavioural characteristics. The multimodal biometric systems provide an advantage over the conventional Unimodal biometric systems in various ways [2]. The main goals of multi-modal biometrics are to reduce at least one of the following; FAR (False Accept Rate), FRR (False Reject Rate), FTE (Failure to Enrollment rate) and Susceptibility to artefacts or mimics. But it also increases sensor cost, enrollment time, transit time and system development [2], [3]. Multimodal biometrics system acquires the input from one or more sensors measuring two or more different modalities of biometric data.

The fusion in a biometric system is to integrate two different biometric data. The fusion can be applied at a various level of the biometric system: sensor level, feature level, score level, rank level, and decision level. In this paper, the features are combined at the feature level. The Histogram of Oriented Gradients (HOG) and the Local Binary Pattern were used for extracting the palm features. The Zernike moments and Local Binary Pattern were used for extracting the face features.

The rest of the will include the related works in section two. The section three will describe the proposed system. The implementation and results will describe in section four. The conclusion will be in section five.

\section{RELATED WORKS}

Yao Fu et al. [4] Proposed two steps for fusion the palmprint and face at the feature level: firstly, since the huge different between the face and palm then it normalized their features as a certain range. Secondly, utilized User-specific weighting rule, where the weights of palmprint are varies from 0.1 to 0.9 , and the weights of the face are varied from 0.9 to 0.1 . Then selected the weight based on the highest recognition rate of all pairs weights of palmprint and face varies weight.

S Noushath et al. [5] proposed fusion of face and palmprint at the four levels and each level had difference techniques: at the sensor level used wavelets based image fusion scheme, at the feature level used few normalization techniques, at the score level used some rules of fusion such as sum, max and min rule to combine the matching score, finally at the score level used a logical AND \& OR operator. After that, they compare the result of each level. Wei Jai et al. [11] proposed the Histogram of Oriented Lines (HOL) for the palmprint recognition along with illumination, rotation variance and translation

\section{PROPOSED SYSTEM}

In this paper, the face and the palmprint features were fused in the feature vector level. The Histogram of Gradient Oriented (HOG) and Local Binary Pattern (LBP) were applied to extract the features of palm. The Local Binary Pattern (LBP) and Zernike moments were applied to extract the face features.

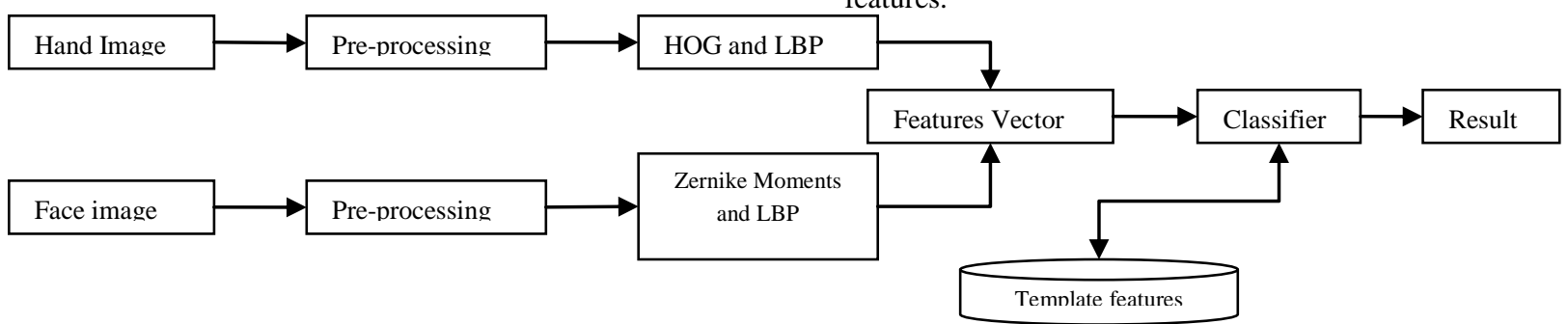

Fig.1. The Proposed System 


\subsection{Pre-processing}

In the case of palmprint, the Region of Interest was extracted by using several steps which are proposed in the previous research [6]. The steps to find the ROI of palm as following

Step.1. Segmented the hand region by using the thresholding or the Skin-color segmentation.

Step.2. Detected the reference points (the root of the fingers) by using the Valley Detection algorithm [7].

Step.3. Establish Coordinate: based on the reference points, the coordinate of the palm is established with help of some mathematics. And this coordinate should be a fixed for all the input images.

Step.4. ROI Extraction: the final step is rotated and extracted the coordinate of palm in a fixed size image. This fixed image is called as Region of Interest (ROI) as shown in the Fig.2.
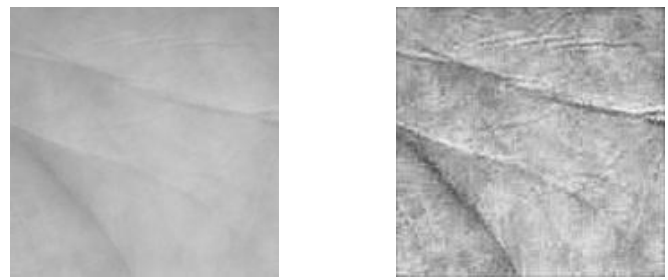

Fig.2. The ROI of Palmprint and the Enhancement

Further, the intensity contrast of ROI images was enhanced by applying the Contrast-Limited Adaptive Histogram Equalization (CLAHE) [8]. Then, the ROI images of palm were resized into $64 \times 64$, Where the face images were resized into $32 \times 32$.

\subsection{Feature Extraction}

The palm and face are having different features, so the Local Binary Pattern is used to extract the texture features of palm and face. In the case of palm, the Sobel and Gaussian filters were applied for increasing the accuracy of features extraction techniques.

\subsubsection{Local Binary Pattern}

The Local Binary Pattern is to compute the relative between the pixels [6], [9]. So, it is compared the central pixel against the 8-neighbor as showing in the Fig.3. If the central pixel value is lower than the neighbor value, then it is assigned as zero. And if the value of neighbor is lower or equal to the central pixel, then it is assigned as one.

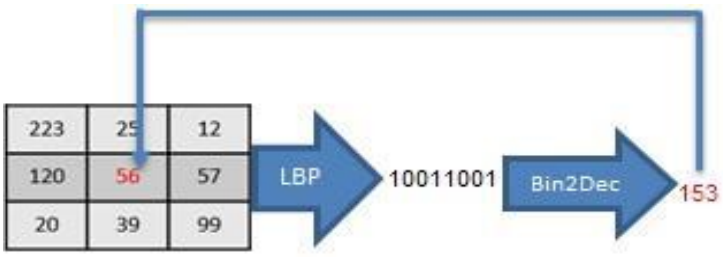

Fig.3. Computing the Local Binary Pattern

Further, the mean and standard deviation were computed on the LBP of palm and face images. The mean and standard deviation equations as follows: -

$$
S=\sqrt{\frac{1}{N-1} \sum_{i=1}^{N}\left|A_{i}-\mu\right|^{2}}
$$

$$
\mu=\frac{1}{N} \sum_{i=1}^{N} A_{i} .
$$

Where. The $S$ and $\mu$ are divided by 255 for making the feature values in the range between 0 and 1 .

\subsubsection{Histogram of Oriented Gradient}

In this paper, the combined of Discrete Cosine Transform (DCT) and the Histogram of Oriented Gradient (HOG) [10] were proposed for extracting the palm features. The DCT is used to extract the edges from the image. So, the DCT was applied in the palm images. After that, the Histogram of Oriented Gradient is applied on the DCT output.

The Histogram of Oriented Gradient steps as following [10], [11]: -

Step.1. divide the palm image into cells and the size of each cell is $8 \times 8$ pixels.

Step.2. each $2 \times 2$ cells will be constructed as Block along with $50 \%$ overlapping as showing the Fig.2. The total number of Blocks in each palm image is $7 \times 7=49$ Blocks.

Step.3. the direction of gradient orientation range $\left(0-180^{\circ}\right)$ will be divided into 9 bins. The gradient magnitude and orientation are computed by the following equations: -

$$
\begin{aligned}
& d x=I(x+1, y)-I(x-1, y) \\
& d y=I(x, y+1)-I(x, y-1) \\
& m(x, y)=\sqrt{d x^{2}+d y^{2}}
\end{aligned}
$$

Step.4. calculate the histogram in the cells with the respect to the bins

Total no of features $=\mathrm{NB} \times \mathrm{CB} \times \mathrm{P}$

Where, NB is the total number of blocks in the palm image. $\mathrm{CB}$ is the cells number in each block. $\mathrm{P}$ is the bins Orientation which is assigned by 9 . From the equation 3 , the total number of $\mathrm{HOG}$ features are $49 \times 4 \times 9=1764$.

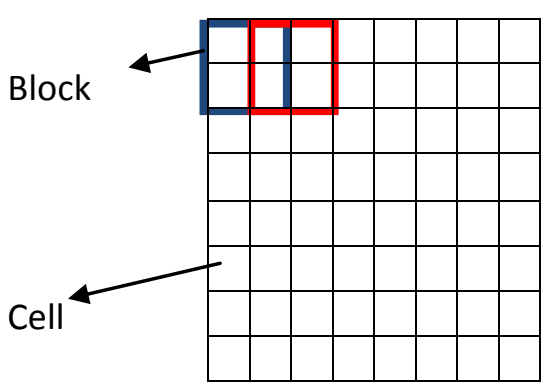

Fig.4. Example of Blocks and Cells in the $64 \times 64$ image

\subsubsection{Gabor and Zernike Moments}

To extract the texture features of face, the combined of Gabor filter and Zernike Moments were proposed [12]. The Gabor filters are widely used in the edge detection, texture analysis, feature extraction and so on. The equations of Gabor filter are shown below: -

$$
\begin{aligned}
& G(x, y, \theta, \lambda, \varphi, \sigma, \gamma)=e^{-\left(\frac{x^{2}+y^{2} y^{2}}{2 \sigma^{2}}\right)} e^{i\left(2 \pi \frac{z^{\prime}}{\lambda}+\emptyset\right)} \\
& x^{\prime}=x \cos (\theta)+y \sin (\theta) \\
& y^{\prime}=-x \sin (\theta)+y \cos (\theta)
\end{aligned}
$$




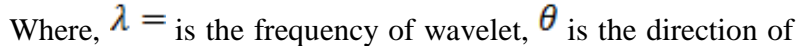
the wavelet. The scale is $\sigma$ and $\varphi$ is the phase. The $\gamma$ is the aspect ratio of the Gaussian. 8 orientations and 5 scales were used for this experiment. So, the face image was divided into 40 sub-images. The Zernike moment is applied on the subimages. The 4 orders and 4 Zernike features were selected from the sub images. Therefore, the total number of Zernike feature is $40 \times 4=160$ features.

The total features of the palm and face are $60+122+1764+160=2106$

Where, 60 features for Local Binary Pattern of the face. 122 features for the Local Binary Pattern of the palm. 1764 features for the Histogram of Oriented Gradient of the palm. 160 features for the Zernike Moments of the face.

\subsection{Classification}

The classification is used to evaluate the feature vectors. In this paper, different classifications were applied such as Support Vector Machine (SVM), k-nearest neighbors (KNN), Linear Discriminant etc. as showing on the table 1. Those classifications are available in the classify learner application in MATLAB.

\subsection{Database}

Two databases were used for this experiment. The ORL database [13] is contained 400 grey-face images which have been collected on April 1992-1994 from 40 people. The dimensionality of each image is $92 \times 112.8$ images from each 24 persons were select for this experiment. So, the total of face image is $8 \times 24=192$ images. The second database is CASIA-Palmprint image database [14], and it contained 5502 palmprint images which are captured from 312 subjects. All images are grey-level JPEG files. But in this experiment, 192 image were selected from the left hand.

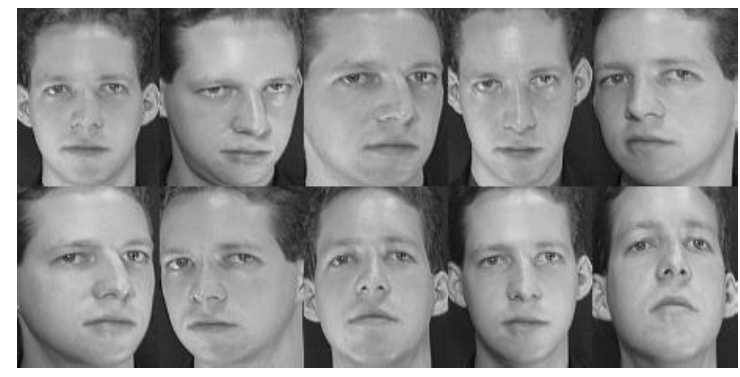

Fig.5. Single subject Face Images of ORL

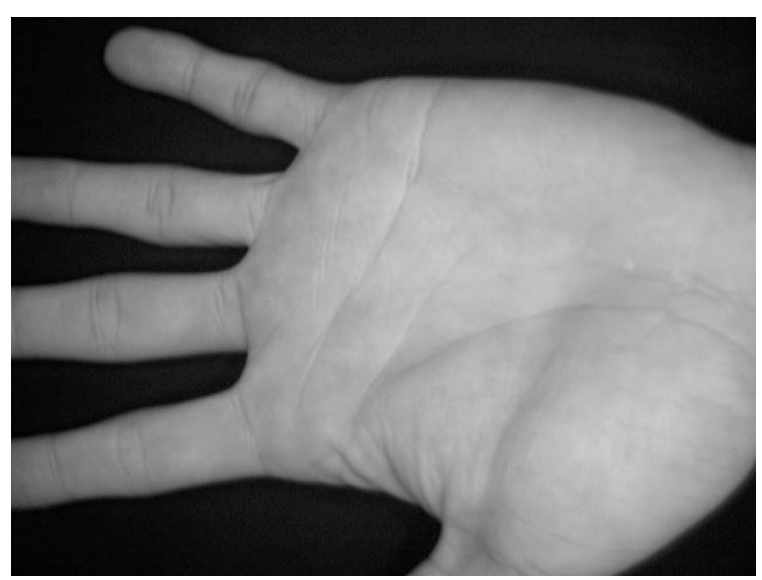

Fig.6. Single Sample of Palm Images of CASIA Database

\section{IMPLEMENTATION AND RESULTS}

The implementation was programed on the MATLAB R2013a and MATLAB 2017a. The system configurations are i7 CPU $2.50 \mathrm{GHz}(8 \mathrm{CPU})$, Windows 10 and the RAM is $8 \mathrm{~GB}$. The Classification Learner Application is used which is available on MATLAB. The PCA tool was used to reduce the features in the e system. The training and testing are divided $50 \%$ for both.

Table 1 Results of Multimodal of palmprint and face

\begin{tabular}{|c|c|c|c|}
\hline Classification & $\begin{array}{c}\text { No of } \\
\text { features }\end{array}$ & Time & Accuracy \\
\hline \multirow{2}{*}{ Complex tree } & all & 15.844 & $45.8 \%$ \\
\hline & 54 PCA on & 157.74 & $56.3 \%$ \\
\hline \multirow{2}{*}{$\begin{array}{c}\text { Linear } \\
\text { Discriminant }\end{array}$} & All & 6.1804 & $100 \%$ \\
\hline & 54 PCA on & 150.25 & $99 \%$ \\
\hline \multirow{2}{*}{ Linear SVM } & All & 38.138 & $100 \%$ \\
\hline & 54 PCA on & 172.87 & $99 \%$ \\
\hline \multirow{2}{*}{ Quadratic SVM } & All & 43.399 & $100 \%$ \\
\hline & 54 PCA on & 186.01 & $96.9 \%$ \\
\hline \multirow{2}{*}{ Cubic SVM } & All & 51.832 & $100 \%$ \\
\hline & 54 PCA on & 215.5 & $94.8 \%$ \\
\hline \multirow{2}{*}{$\begin{array}{c}\text { Fine Gaussian } \\
\text { SVM }\end{array}$} & All & 48.476 & $4.2 \%$ \\
\hline & 54 PCA on & 209.64 & $95.8 \%$ \\
\hline \multirow{2}{*}{$\begin{array}{c}\text { Medium Gaussian } \\
\text { SVM }\end{array}$} & All & 66.238 & $97.9 \%$ \\
\hline & 54 PCA on & 203.59 & $95.8 \%$ \\
\hline \multirow{2}{*}{$\begin{array}{c}\text { Coarse Gaussian } \\
\text { SVM }\end{array}$} & All & 71.56 & $100 \%$ \\
\hline & 54 PCA on & 195.72 & $95.8 \%$ \\
\hline \multirow{2}{*}{ Fine KNN } & All & 51.176 & $100 \%$ \\
\hline & 54 PCA on & 188.52 & $96.9 \%$ \\
\hline \multirow{2}{*}{ Medium KNN } & All & 54.349 & $92.7 \%$ \\
\hline & 54 PCA on & 247.37 & $84.4 \%$ \\
\hline \multirow{2}{*}{ Cosine KNN } & All & 52.372 & $95.8 \%$ \\
\hline & 54 PCA on & 239.96 & $93.8 \%$ \\
\hline \multirow{2}{*}{ Cubic KNN } & All & 55.876 & $91.7 \%$ \\
\hline & 54 PCA on & 234.37 & $71.9 \%$ \\
\hline \multirow{2}{*}{ Weighted KNN } & All & 55.206 & $96.9 \%$ \\
\hline & 54 PCA on & 229.03 & $93.8 \%$ \\
\hline \multirow{2}{*}{$\begin{array}{c}\text { Ensemble (Boosted } \\
\text { Trees) }\end{array}$} & All & 531.06 & $72.9 \%$ \\
\hline & 54 PCA on & 222.98 & $85.4 \%$ \\
\hline \multirow{2}{*}{$\begin{array}{c}\text { Ensemble (Bagged } \\
\text { Trees) }\end{array}$} & All & 65.955 & $92.7 \%$ \\
\hline & 54 PCA on & 218.19 & $90.6 \%$ \\
\hline \multirow{2}{*}{$\begin{array}{c}\text { Ensemble } \\
\text { (Subspace } \\
\text { Discriminant) }\end{array}$} & All & 80.449 & $99 \%$ \\
\hline & $54 \mathrm{PCA}$ on & 243.03 & $99 \%$ \\
\hline \multirow{2}{*}{$\begin{array}{c}\text { Ensemble } \\
\text { (Subspace KNN) }\end{array}$} & All & 71.855 & $100 \%$ \\
\hline & 54 PCA on & 206.93 & $100 \%$ \\
\hline
\end{tabular}


From the above table 1, the Principal Component Analysis (PCA) tool is used to reduce the number of features. So, the number of features after applying the PCA tool are 54 features. The Complex Tree classification and the Fine Gaussian SVM were failed in this system. Where, the accuracy in Linear Discriminant (LD) classification is $100 \%$ and the classification time is 6.1804 seconds. When the features were reduced by using PCA, the accuracy is $99 \%$ for the Linear Discriminant (LD) classification, but the classification time was increasing up to 150.25 seconds. In the Linear SVM, the accuracy was $100 \%$ and the classification time 38.138 seconds. When the features were reduced by using PCA, the accuracy is $99 \%$ and the classification time was increasing also into 172.87 seconds. From the whole table 1, after applying the PCA the time is increasing, while the features are reducing. In the LD, Linear SVM, Quadratic SVM Cubic SVM, Coarse Gaussian SVM, Fine $\mathrm{KNN}$ and Ensemble (Subspace $\mathrm{KNN}$ ) are given the $100 \%$ accuracy.

\section{CONCLUSION}

This paper presents the experimental evaluation of the multimodal recognition system of the palmprint and face. 192 images were selected from each database (ORL and CASIA). The region of interest of palm was extracted and resized into $64 \times 64$. Where, the face images were resized into $32 \times 32$. The Local Binary Pattern and Histogram of Oriented Gradient were used for extracting the features of palm. The Local Binary Pattern and Zernike Moments were used for extracting the features of the face. The length of the feature vector is 2106 but it was reduced by using the PCA tool to 54 . The Linear Discriminant classification is given the best accuracy and time as well.

\section{ACKNOWLEDGMENTS}

This work is sponsored by SAP DRS-I (No: F.3-52/2011), UGC New Delhi, India.

\section{REFERENCES}

[1] Anil K.jain,Arun A.Ross,Karthik Nandakumar, "introduction to biometrics", Springer Sciences + Business Media, LLC, 233 Spring Street, New York,NY 10013, USA , July 2011

[2] Ashish Mishra, "Multimodal biometrics it is : need for future system", International journal of computer applications(0975-8887) volume 3-No.4,june 2010

[3] Teddy ko, "multimodal biometric identification for large user population using fingerprint, face and iris recognition", IEEE Proceedings of the 34th Applied Imagery and Pattern Recognition Workshop (AIPR05),2005
[4] Y. Fu, Z. Ma, M. Qi, J. Li, X. Li, and Y. Lu, "a novel user-specific face and palmprint feature level fusion", IEEE second international symposium on intelligent information technology application, 987-0-7695-34978/08, Chine 2008

[5] S Noushath, M. Imran, A. Rao and H. Kumar G "multimodal biometric fusion of face and palmprint at various levels", IEEE

[6] Akram Alsubari and R. J. Ramteke, "Extraction of palmprint texture features using combined DWT-DCT and local binary pattern,", IEEE 2nd International Conference on Next Generation Computing Technologies (NGCT), Dehradun, 2016, pp. 748-753.

[7] Goh Kah Ong Michael, Tee Connie and Andrew Beng Jin Teoh, "Touch-less Palmprint Biometrics: Novel Design and Implementation", ELSEVIER Image and Vision Computing 26 (2008) 1551-1560

[8] Zuiderveld and Karel, "Contrast Limited Adaptive Histogram Equalization", Graphic Gems IV. San Diego: Academic Press Professional, 1994. 474-485

[9] Akram Alsubari, D. N. Satange and R. J. Ramteke, "Facial Expression Recognition using Wavelet Transform and Local Binary Pattern", IEEE 2nd International Conference for Convergence in Technology (I2CT), Pune, 2017

[10] Navneet Dalal and Bill Triggs, "Histogram of Oriented Gradients for Human Detection", IEEE Computer Society Conference on Computer Vision and Pattern Recognition (CVPR'05),1063-6919/05, 2005

[11] Wei Jia, Rong-Xiang Hu, Ying-Ke Lei, Yang Zhao, and Jie Gui, "Histogram of Oriented Lines for Palmprint Recognition", IEEE Transaction on Systems, Man, and Cybernetics: Vol. 44, No. 3, March 2014

[12] D. N. Satange, Akam Alsubari and R. J. Ramteke, "Composite Feature Extraction based on Gabor and Zernike Moments for Face Recognition", IOSR-JCE, International Conference on Recent Advance in Computer Science, Engineering and Technology, Aurangabad, PP 17-23, Feb 2017

[13] Olivetti Research Laboratory (ORL) Face Database is available on the following link: http://www.cl.cam.ac.uk/research/dtg/attarchive/facedata base.html

[14] CASIA Palmprint Database is available on this link: http://www.biometrics.idealtest.org 